\title{
ORIGINAL ARTICLE \\ Observations in the diagnosis of cervical myelopathy in patients suffering from diabetes mellitus
}

\author{
M Okada, A Minamide, M Yoshida, M Kawakami, Y Enyo and T Endo
}

Objectives: To identify any observations that could aid in the diagnosis of cervical myelopathy in patients suffering from diabetes mellitus (DM). We compared the preoperative neurological findings in patients with cervical myelopathy among non-diabetics, mild diabetics and severe diabetics.

Study Design: A retrospective comparative study.

Setting: Department of Orthopaedic Surgery, Wakayama Medical University, Japan.

Methods: We retrospectively reviewed 111 patients who had undergone laminoplasty for cervical compressive myelopathy: 56 without DM (group N), 26 with mild diabetes (group M) and 29 with severe diabetes (group S). We regarded the patients as severe diabetics when they had a history of one or more of following; (a) more than 10 years of medication; (b) more than 7.0\% $\mathrm{HbA1c}$; (c) diabetic retinopathy; and (d) delayed conduction velocity of peripheral nerves. For preoperative neurological assessment we compared the following among the three groups: (1) the 10 s test whereby the myelopathy in the hand was quantified; (2) sensory disturbance; (3) deep tendon reflexes; (4) Hoffmann's, Trömner's and Babinski's reflexes; and (5) bladder dysfunction.

Results: There was no significant difference preoperatively in the $10 \mathrm{~s}$ test between the groups. Deep tendon reflexes were significantly decreased in group S. There were no significant differences in sensory disturbance and bladder dysfunction. Although Hoffmann's and Trömner's reflexes significantly disappeared in group S, there was no significant difference in positivity of Babinski's reflex between the groups.

Conclusions: The $10 \mathrm{~s}$ test and Babinski's reflex are helpful for the diagnosis of cervical myelopathy in patients suffering from DM. Spinal Cord (2012) 50, 878-881; doi:10.1038/sc.2012.56; published online 15 May 2012

Keywords: cervical myelopathy; diabetes mellitus; Babinski's reflex

\section{INTRODUCTION}

Diabetes mellitus (DM) is a chronic systemic disease, which is characterized by hyperglycaemia. Long-term morbidity of DM results in peripheral neuropathy, diabetic neuropathy, which causes sensory disturbance in the limbs and a failure or absence of deep tendon reflexes. The autonomic nerve system is also affected by DM and the disorder leads to bladder-bowel dysfunction.

Meanwhile, cervical myelopathy also shows sensory disturbance in the limbs, and bladder dysfunction. Most of severe-diabetic patients already have sensory disturbance in the limbs, and bladder dysfunction, therefore these findings cannot be used to clarify the presence of cervical myelopathy in patients suffering from DM. Furthermore, deep tendon hyperreflexias that are the typical signs for cervical myelopathy will not be apparent in patients with DM (as per above). Thus, the diagnosis of cervical myelopathy in patients suffering from DM is difficult.

Some previous reports described the differences in the neurological findings for cervical myelopathy between diabetic and non-diabetic patients. $^{1,2}$ However, none of these previous reports provide information regarding signs that could help aid the diagnosis of cervical myelopathy in patients with DM. In these previous studies patients with mild and severe degree of DM were mixed together.

We therefore carried out a study to compare the preoperative neurological findings in patients with cervical myelopathy without diabetes; with mild diabetes; and with severe diabetes, in order to identify any features/observations that could aid in the diagnosis of cervical myelopathy in patients suffering from diabetes.

\section{PATIENTS AND METHODS}

We retrospectively reviewed 111 patients who had undergone laminoplasty for cervical compressive myelopathy from 2002-2009 at our institute. There were 55 patients with DM and 56 without DM (group N). The 55 patients with diabetes consisted of 26 mild diabetics (group M) and 29 severe diabetics (group S). We regarded the patients as severe diabetics when they had a history of one or more of the following: (a) more than 10 years of medication; (b) more than 7.0\% HbAlc; (c) diabetic retinopathy; and (d) delayed conduction velocity of peripheral nerves. We chose $7.0 \%$ for the criterion of $\mathrm{HbAlc}$ because previous reports suggested that chronic complications due to DM occurred when HbAlc was more than 7.0\%.3.,4 We chose more than 10 years of medication based on the report that the incidence of chronic complications begin to increase after 10 years. ${ }^{3,4,5}$ The reason we chose (c) and (d) is that the presence of diabetic retinopathy or delayed conduction velocity of peripheral nerves shows that a patient is not a mild diabetic.

Sex, age and cause of cervical myelopathy are shown in Table 1. There were no significant differences in the sex, age, cause of cervical myelopathy, level of compressive lesion in the spinal cord, surgical level and morbidity period among the three groups. Patients who had rheumatic cervical lesions, pyogenic spondylitis, destructive spondyloarthropathy and traumas were excluded. We also excluded patients who were diagnosed with a disease that modified cervical myelopathy, such as Parkinson's disease. 
Table 1 Sex, age and cause of cervical myelopathy in the three groups of patients

\begin{tabular}{lccc}
\hline Group & $N$ & $M$ & $S$ \\
\hline$N$ & 56 & 26 & 29 \\
Sex & & & $21(72.4 \%)$ \\
Male & $32(57.1 \%)$ & $6(23.1 \%)$ & $8(27.6 \%)$ \\
Female & $24(42.9 \%)$ & 48 ) & \\
Age & $40-85$ (average 59.1) & 48 (average 64.4) & $48-86$ (average 66.4) \\
(years) & & & \\
Diagnosis & & $14(53.8 \%)$ & $17(58.6 \%)$ \\
CSM & $32(57.1 \%)$ & $10(38.5 \%)$ & $10(34.5 \%)$ \\
OPLL & $21(37.6 \%)$ & $2(7.7 \%)$ & $2(6.9 \%)$ \\
CDH & $3(5.4 \%)$ & & \\
\hline
\end{tabular}

Abbreviations: $\mathrm{CDH}$, cervical disc herniation; CSM, cervical spondylotic myelopathy; OPLL, ossification of the posterior longitudinal ligament.

Group N: non-diabetic patients with cervical myelopathy.

Group M: mild-diabetic patients with cervical myelopathy.

Group S: severe-diabetic patients with cervical myelopathy.

We evaluated the following for preoperative neurological findings: (1) the $10 \mathrm{~s}$ test $^{6}$; (2) sensory disturbance; (3) deep tendon reflexes; (4) eponymous reflexes (Hoffmann, Trömner and Babinski reflexes); and (5) bladder dysfunction. The $10 \mathrm{~s}$ test is the test used to measure the frequency of finger grip and release in $10 \mathrm{~s}$. The myelopathy in the hand is quantified by the test. A number of 20 or less is interpreted as 'clumsy'. For comparison of sensory disturbance, we used the lowest score of the pin-prick test when applied to the trunk and extremities. Hoffmann and Trömner reflexes are known as the finger flexor and extensor reflex, respectively. The tests involve tapping the nail or flicking the terminal phalanx of the middle finger. A positive response is seen with flexion of the terminal phalanx of the thumb. The tests verify the presence or absence of problems in the corticospinal tract. Babinski reflex is known as one of the plantar reflex elicited when the sole of the foot is stimulated with a blunt instrument. An upward response of the hallux is known as Babinski sign. The presence of the Babinski sign can indicate upper motor neuron lesion constituting damage to the corticospinal tract. The (Japanese Orthopaedics Association Score) JOA scoring system $^{8}$ (as proposed by the Japanese Orthopaedic Association) was also assessed pre- and postoperatively. The scoring system is commonly used in Japan to indicate the severity in cervical myelopathy. The total score for a healthy individual is 17. The scoring system covers the following: (1) motor function at upper and lower extremity; (2) sensory function at upper and lower extremity and trunk; and (3) bladder function (Table 2).

The results obtained for the preoperative $10 \mathrm{~s}$ test and sensory disturbance were analysed by analysis of variance between the groups. Analysis of variance was also used for statistical comparison of the change in JOA score among the groups. The Mann-Whitney $U$-test was used for statistical analysis in deep tendon reflexes, eponymous reflexes and bladder dysfunction. A probability level of $<0.05$ was considered significant.

Cervical French-door laminoplasty with reattachment of the spinous process and extensor musculatures ${ }^{7}$ was carried out for decompression of the spinal cord. In the DM patients that showed hypo- or normoreflexia and negative Babinski's reflex, all of them showed myelopathic hand as evaluated by the $10 \mathrm{~s}$ test. Moreover skillfulness at upper extremities and gait disturbance were evaluated using the JOA scoring system. MRI and myelography were also performed for the diagnosis of spinal cord compression. It was therefore considered that the diagnosis of myelopathy should be reasonable even in the diabetic groups.

\section{RESULTS}

The follow-up period for postoperative assessment was at least 6 months in all groups and the mean follow-up period was 26.6, 34.5 and 32.2 months in groups $\mathrm{N}, \mathrm{M}$ and S, respectively.

There was no significant difference preoperatively in the $10 \mathrm{~s}$ test between the three groups (Group N 13.6 \pm 5.3 times, Group M $14.1 \pm 4.7$ times and Group S $14.9 \pm 4.6$ times). This indicates that the patients in group N, M and S were similarly 'clumsy'.

\section{Table 2 Japanese Orthopaedics Association Score (JOA score)}

\section{Japanese Orthopaedics Assocation Score}

\section{Motor function of upper extremity \\ 0: Unable to eat with either spoon or chopsticks \\ 1: Possible to eat with spoon, but not chopsticks \\ 2: Possible to eat with chopsticks, but inadequate \\ 3: Possible to eat with chopsticks, but awkward \\ 4: Normal}

II Motor function of lower extremity

0: Impossible to walk

1: Need cane or aid on flat ground

2: Need cane or aid only on stairs

3: Possible to walk without cane or aid, but slow

4: Normal

III Sensory deficit

(A) Upper extremity

0 : Apparent sensory loss

1: Minimal sensory loss

2: Normal

(B) Lower extremity

0 : Apparent sensory loss

1: Minimal sensory loss

2: Normal

(C) Trunk

0 : Apparent sensory loss

1: Minimal sensory loss

2: Normal

IV Sphincter dysfunction

0 : Complete urinary retention

1: Severe disturbance

2: Mild disturbance

3: Normal

There was no significant difference in sensory disturbance between the three groups (Group N $4.0 \pm 2.9$, Group M $4.5 \pm 2.1$ and Group S $3.8 \pm 2.1)$

As expected, all of deep tendon reflexes were significantly decreased in group $\mathrm{S}$ as compared with groups $\mathrm{N}$ and $\mathrm{M}$ (Table 3 ).

Interestingly among the eponymous reflexes, although Hoffmann's and Trömner's reflexes significantly disappeared in group $S$ as compared with groups $\mathrm{N}$ and $\mathrm{M}$, there was no significant difference in positivity of Babinski's reflex between the three groups (Table 4).

There was no significant difference in bladder dysfunction between the three groups. There was no significant difference in the change for the result of the $10 \mathrm{~s}$ test between pre and postop between the three groups. Also there was no significant difference in the change of JOA score of motor function at upper extremities (total score is 4) between pre and postop between the three groups (Figures 1 and 2).

\section{DISCUSSION}

The important results to come from this study are: (1) there was no significant difference in the preoperative $10 \mathrm{~s}$ test between the three groups; (2) Although Hoffmann's and Trömner's reflexes significantly disappeared in group $\mathrm{S}$ as compared with groups $\mathrm{N}$ and $\mathrm{M}$, there was no significant difference in positivity of Babinski's reflex between the three groups.

The lack of difference in the results of the preoperative $10 \mathrm{~s}$ test between the three groups indicates that clumsiness appears similarly in patients with cervical myelopathy, as well as with and without DM. In 
Table 3 Comparison of deep tendon reflexes

\begin{tabular}{|c|c|c|c|}
\hline & Decrease & Normal & Increase \\
\hline \multicolumn{4}{|l|}{ BTR } \\
\hline Group N & $4(7.1 \%)$ & 20 (35.7\%) & $32(57.1 \%)$ \\
\hline Group M & $3(11.5 \%)$ & $11(42.3 \%)$ & $12(46.2 \%)$ \\
\hline Group S* & 11 (37.9\%) & 7 (24.1\%) & 11 (37.9\%) \\
\hline \multicolumn{4}{|l|}{ BrTR } \\
\hline Group N & $2(3.6 \%)$ & $14(25.0 \%)$ & 40 (71.4\%) \\
\hline Group M & 2 (7.7\%) & 11 (42.3\%) & $13(50.0 \%)$ \\
\hline Group $\mathrm{S}^{*}$ & $8(27.6 \%)$ & 11 (37.9\%) & $10(34.5 \%)$ \\
\hline \multicolumn{4}{|l|}{ TTR } \\
\hline Group N & $4(7.1 \%)$ & $16(28.6 \%)$ & $36(64.3 \%)$ \\
\hline Group M & $3(11.5 \%)$ & 9 (34.6\%) & $14(53.8 \%)$ \\
\hline Group S* & $8(27.6 \%)$ & $13(44.8 \%)$ & $8(27.6 \%)$ \\
\hline \multicolumn{4}{|l|}{ PTR } \\
\hline Group N & $1(1.8 \%)$ & $3(5.4 \%)$ & 52 (92.9\%) \\
\hline Group M & 5 (19.2\%) & 7 (26.9\%) & $14(53.8 \%)$ \\
\hline Group S* & $12(41.4 \%)$ & $6(20.7 \%)$ & $11(37.9 \%)$ \\
\hline \multicolumn{4}{|l|}{ ATR } \\
\hline Group N & $3(5.4 \%)$ & $8(14.3 \%)$ & $45(80.4 \%)$ \\
\hline Group M & 7 (26.9\%) & 7 (26.9\%) & $12(46.2 \%)$ \\
\hline Group S* & $20(69.0 \%)$ & 5 (17.2\%) & 4 (13.8\%) \\
\hline
\end{tabular}

Abbreviations: ATR, achilles tendon reflex; BrTR, Brachioradialis tendon reflex; BTR, Biceps tendon reflex; PTR, Patellar tendon reflex; TTR, Triceps tendon reflex. ${ }^{*} P<0.05$.

Table 4 Comparison of eponymic reflexes

\begin{tabular}{llr}
\hline & Positive & Negative \\
\hline $\begin{array}{l}\text { Hoffmann } \\
\text { Group N }\end{array}$ & $50(89.3 \%)$ & $6(10.7 \%)$ \\
Group M & $20(76.9 \%)$ & $6(23.1 \%)$ \\
Group S* & $11(37.9 \%)$ & $18(62.1 \%)$ \\
Trömner & & \\
Group N & $50(89.3 \%)$ & $6(10.7 \%)$ \\
Group M & $20(76.9 \%)$ & $6(23.1 \%)$ \\
Group S* & $11(37.9 \%)$ & $18(62.1 \%)$ \\
Babinski & & $24(42.9 \%)$ \\
Group N & $32(57.1 \%)$ & $13(50.0 \%)$ \\
Group M & $13(0.0 \%)$ & $10(34.5 \%)$ \\
Group S & $19(65.5 \%)$ & \\
\hline
\end{tabular}

$\left({ }^{*} P<0.05\right)$.

other words, diabetic neuropathy does not affect clumsiness caused by cervical myelopathy. Some textbooks also say that diabetic neuropathy does not induce myelopathy. ${ }^{5}$ So, this indicates that the appearance of a myelopathic hand directly means the presence of myelopathy, with or without diabetic neuropathy. This result therefore suggests that the $10 \mathrm{~s}$ test by which 'myelopathy' was quantified is a helpful observation to make in the diagnosis for cervical myelopathy in patients suffering from DM. In addition, we should be able to expect that decompression surgery would produce an improvement postoperatively in terms of clumsiness as diabetic neuropathy does not affect clumsiness (mentioned in Results and shown in Figures 1 and 2).

As expected, all of deep tendon reflexes were significantly decreased in group $\mathrm{S}$ as compared with group $\mathrm{N}$ and $\mathrm{M}$. This indicates that in the presence of severe DM, hyperreflexia caused by cervical myelopathy are not apparent because of the presence of peripheral

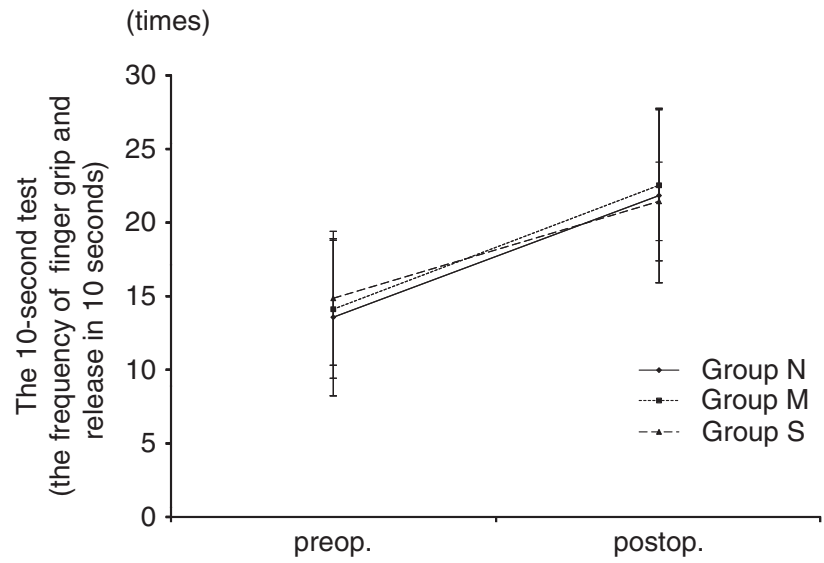

Figure 1 Comparison of the change in the results for the $10 \mathrm{~s}$ test between pre and postop among the three groups. There is no significant difference in the change of $10 \mathrm{~s}$ test between pre and postop among the three groups.

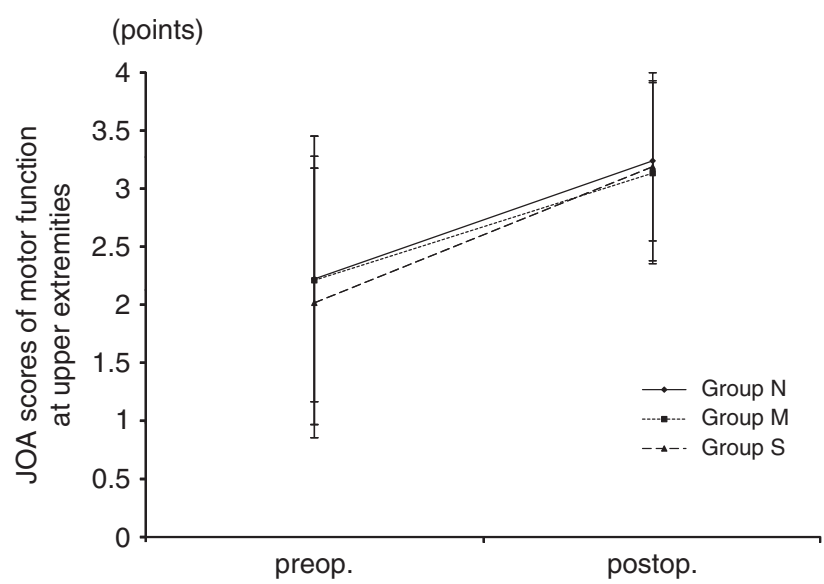

Figure 2 Comparison of the change in Japanese Orthopaedics Association Score (JOA) scores of motor function at upper extremities (total score is 4) between pre and postop among the three groups. There is no significant difference in the change of JOA score of motor function at upper extremities between pre and postop among the three groups.

neuropathy. Therefore, deep tendon reflexes cannot help in making the diagnosis for cervical myelopathy in patients suffering from DM. Interestingly among the eponymous reflexes, while Hoffmann's and Trömner's reflexes significantly disappeared in severe-diabetic patients as compared with non- and mild-diabetic patients, there was no significant difference in positivity of Babinski's reflex between severe-, mild- and non-diabetic patients. This indicates that even if all of deep tendon reflexes are decreased by DM, Babinski's reflex can be apparent in severe-diabetic patients similarly with non-diabetic patients. Hoffmann's and Trömner's reflexes are often considered the upper limb equivalent of the Babinski's reflex, because these reflexes indicate upper motor neuron dysfunction. However, Hoffmann's and Trömner's reflexes mechanistically differ from the Babinski's reflex; Hoffmann's and Trömner's reflexes are a kind of stretch reflex that involves a monosynaptic reflex pathway in Rexed lamina IX of the spinal cord, normally fully inhibited by descending input. ${ }^{9}$ This means the positive Hoffmann's and Trömner's reflexes are caused through the same physiological mechanism as deep tendon hyperreflexia. Thus, these two eponymous reflexes were decreased because of diabetic neuropathy. On the other hand, Babinski's reflex really reflects a pathological reflex and long tract sign. The pathways involved in 
Babinski's reflex are more complicated than those in Hoffmann's and Trömner's reflexes and different sorts of lesions may interrupt them. Taken together, of the eponymous reflexes only Babinski's reflex is helpful in making a diagnosis for cervical myelopathy in patients suffering from DM. Although the results of some previous reports suggest that DM does not have any significant affect on the prevalence of any myelopathic signs, ${ }^{1,2}$ these results, which differ from our results, might have come from a small number of the diabetic patients or the mixture of patients with mild and severe DM.

There were no significant differences in both sensory disturbance and bladder dysfunction among the three groups. This suggests that we were not able to detect the presence of cervical myelopathy in DM patients by either the degree of sensory disturbance or the presence of bladder dysfunction. Thus, both sensory disturbance and bladder dysfunction are not helpful in making the diagnosis for cervical myelopathy in patients suffering from DM. These indicate that we should not expect a postoperative improvement in either sensory disturbance or bladder dysfunction by decompression surgery.

In conclusion, the $10 \mathrm{~s}$ test and Babinski's reflex are helpful for the diagnosis of cervical myelopathy in patients suffering from DM.

\section{CONFLICT OF INTEREST}

The authors declare no conflict of interest

1 Houten JK, Noce LA. Clinical correlations of cervical myelopathy and the Hoffmann sign. J Neurosurg Spine 2008; 9: 237-242.

2 Rhee JM, Heflin JA, Hamasaki T, Freedman B. Prevalence of physical signs in cervical myelopathy: a prospective, controlled study. Spine 2008; 34: 890-895.

3 Minuk H. Diabetes mellitus: new guidelines. J Insur Med 2000; 32: 269-270.

4 Resnick HE, Foster GL, Bardsley J, Ratner RE. Achievement of American Diabetes Association clinical practice recommendations among U.S. adults with diabetes, 1999-2002: the National Health and Nutrition Examination Survey. Diabetes Care 2006; 29: 531-537.

5 Biessels GJ. Central diabetic neuropathy. In: Gries FA (ed). Textbook of Diabetic Neuropathy. Stuttgart: New York.

6 Ono K, Ebara S, Fuji T, Yonenobu K, Fujiwara K, Yamashita K. Myelopathy hand. New clinical signs of cervical cord damage. J Bone Joint Surg Br 1987; 69: 215-219.

7 Yoshida M, Otani K, Shibasaki K, Ueda S. Expansive laminoplasty with reattachment of spinous process and extensor musculature for cervical myelopathy. Spine 1992; 17: 491-497.

8 Japanese Orthopaedic Association. Scoring system for cervical myelopathy. Nippon Seikeigeka Gakkai Zasshi 1994; 68: 490-503.

9 Harrop JS, Hanna A, Silva MT, Sharan A. Neurological manifestations of cervical spondylosis: an overview of signs, symptoms, and pathophysiology. Neurosurgery 2007; 60: S14-S20.

\section{Editorial Note on: Observations in the diagnosis of cervical myelopathy in patients suffering from diabetes mellitus}

Spinal Cord (2012) 50, 881; doi:10.1038/sc.2012.64; published online 5 June 2012

Editorial Note on: Spinal Cord (2012) 50, 878-881; doi:10.1038/ sc.2012.56; published online 15 May 2012

Cervical myelopathy in patients suffering from diabetes pose a diagnostic challenge to clinicians. Long-term morbidity of diabetes results in peripheral neuropathy and bladder disturbance. Cervical myelopathy also causes sensory disturbance in the limbs and bladder dysfunction. Therefore, the presence of sensory disturbance and bladder dysfunction cannot be used to identify cervical myelopathy in patients suffering from diabetes.

The estimates show an increase in the global prevalence of diabetes for all age groups and especially among people $>65$ years of age. ${ }^{1}$ Cervical spondylotic myelopathy is a degenerative process involving the discs and joints of the cervical spine, which can eventually result in spinal cord compression. The natural history of this disorder is usually slow deterioration in a stepwise manner, with worsening symptoms of gait abnormalities, weakness, sensory changes and often pain. ${ }^{2}$ The prevalence of cervical spondylotic myelopathy increases with increasing age. ${ }^{3}$

Having both diabetes and cervical myelopathy makes it difficult to predict the outcome of surgery. Okada et al. ${ }^{4}$ propose, in this issue, that the 10-s step test and Babinski's reflex are helpful for the diagnosis of cervical myelopathy in patients suffering from diabetes. The 10-s step test is useful as a quantifiable measure of severity in cervical compressive myelopathy and also in determine the effects of decompressive surgical treatment. ${ }^{5}$ They studied the problem by reviewing patients who had undergone laminectomy for cervical compressive myelopathy: one group without diabetes, one with mild diabetes and one with severe diabetes. No statistical difference was found preoperatively between the three groups performing the test, but there was a statistical difference between pre- and post testing in all three groups.

With the increasing prevalence of both diabetes and cervical spondylotic myelopathy, there is a need for tests that may be of help for the diagnosis of cervical spondylotic myelopathy in patients suffering from diabetes, as well as to determine the effects of surgical treatment.

\section{CONFLICT OF INTEREST}

The author declares no conflict of interest.

EM Hagen

Department of Clinical Medicine, Faculty of Medicine and Dentistry, University of Bergen, Bergen, Norway E-mail: ellen.merete.hagen@helse-bergen.no

1 Wild S, Roglic G, Green A, Sicree R, King H. Global prevalence of diabetes: estimates for the year 2000 and projections for 2030. Diabetes Care 2004; 27: 1047-1053.

2 Baron EM, Young WF. Cervical spondylotic myelopathy: a brief review of its pathophysiology, clinical course, and diagnosis. Neurosurgery 2007; 60: S35-S41.

3 Northover JR, Wild JB, Braybrooke J, Blanco J. The epidemiology of cervical spondylotic myelopathy. Skeletal Radiol (e-pub ahead of print 17 March 2012; doi:101007/s00356-012-1388-3).

4 Okada M, Minamide A, Yoshida M, Kawakami M, Enyo Y, Endo T. Observations in the diagnosis of cervical myelopathy in patients suffering from diabetes mellitus. Spinal Cord 2012; 50: 878-881.

5 Yukawa Y, Kato F, Ito K, Horie Y, Nakashima H, Masaaki M et al. 'Ten second step test' as a new quantifiable parameter of cervical myelopathy. Spine 2009; 34: 82-86. 